\title{
Classes of $p$-ideals of Lattice pseudo-Wajsberg Algebras
}

\author{
A. Ibrahim ${ }^{1}$ and M. Indhumathi ${ }^{2}$ \\ ${ }^{1}$ Assistant Professor, $P G$ and Research Department of Mathematics, \\ H. H. The Rajah's College, Pudukkottai-622001, Tamil Nadu, India. \\ ${ }^{2}$ Assistant Professor, Department of Mathematics, \\ Rathnavel Subramaniam College of Arts and Science, \\ Sulur, Coimbatore-641402,Tamil Nadu, India. \\ Email: ${ }^{1}$ ibrahimaadhil@yahoo.com, ${ }^{1}$ dribrahimaadhil@gmail.com, ${ }^{2}$ indumathi@rvsgroup.com.
}

\begin{abstract}
In this paper, we introduce the notions of fuzzy $p$-ideal, anti-fuzzy $p$-ideal, intuitionistic fuzzy $p$-ideal and fuzzy $p$-lattice ideal, anti-fuzzy $p$-lattice ideal and intuitionistic fuzzy $p$-lattice ideal in lattice pseudoWajsberg algebra. Also, we discuss some of their related properties with illustrations.
\end{abstract}

Keywords: Fuzzy subsets; fuzzy $p$-ideal; fuzzy $p$-lattice ideal; anti-fuzzy $p$-ideal; anti-fuzzy $p$-lattice ideal; intuitionistic fuzzy $p$-ideal; intuitionistic fuzzy $p$-lattice ideal.

\section{Mathematics Subject Classification: 03E70, 03E72, 06B10, 06D15.}

\section{INTRODUCTION}

Fuzzy sets are a kind of useful mathematical structure to represent a collection of objects whose boundary is vague. In the traditional fuzzy sets, the membership degrees of elements range over the interval $[0,1]$. The membership degree expresses the degree of belongingness of elements to a fuzzy set. The notion of fuzzy ideal has been introduced in many algebraic structures such as lattice, rings and algebras. Ideal theory is very effective tool for studying various algebraic and logical systems. In order to study the basic logic framework of fuzzy set theory were introduced by Zadeh [15] in 1965.The concept of an anti-fuzzy subgroups of groups was introduced by Biswas[5] in 1990.The concept of intuitionistic fuzzy subset was introduced by Atanassov [3] in 1986, which is a generalization of the notion of fuzzy sets. Fuzzy sets give a degree of membership of an element in a given set, while intuitionistic fuzzy sets give both a degree of membership and a degree of nonmembership to every object. The only constraint on these two degrees is that the sum must smaller than or equal to one. Mordcha jWajsberg [14] introduced the concept of Wajsberg algebras in 1935 and studied by Font, Rodriguez and Torrenns [8]. Also, they [8] defined lattice structure of Wajsberg algebras. Further, they [8] introduced the notion of an implicative filter of lattice Wajsberg algebras and discussed some properties. Pseudo-
Wajsberg algebras are generalizations of Wajsberg algebras. Pseudo-Wajsberg algebras were introduced by Ceterchi Rodica [6] in 2001. Ceterchi Rodica [7] introduced the lattice structure of pseudo-Wajsberg algebras and discussed some results in generalized pseudo-Wajsberg algebras. The authors [9] introduced the notions of PWIideal, pseudo lattice ideal and also the authors [11] introduced the notions of $p$-ideal, $p$-lattice ideal and investigated some properties of lattice pseudoWajsberg algebra. In the present paper, we introduce the notions of fuzzy $p$-ideal, anti-fuzzy $p$-ideal, intuitionistic fuzzy $p$-ideal and fuzzy $p$-lattice ideal, anti-fuzzy $p$-lattice ideal and intuitionistic fuzzy $p$-lattice ideal in lattice pseudoWajsberg algebra. Also, we discuss some of their related properties with illustrations.

\section{PRELIMINARIES}

In this section, we submit some concepts related to lattice pseudo-Wajsberg algebra. Meanwhile, some comments and results are obtained.

Definition 2.1[8]. An algebra $(A, \rightarrow,-, 1)$ with a binary operation " $\rightarrow$ " and a quasi-complement" " is called a Wajsberg algebra if it satisfies the following axioms for all $x, y, z \in A$,

(i) $1 \rightarrow x=x$

(ii) $(x \rightarrow y) \rightarrow y=(y \rightarrow x) \rightarrow x$

(iii) $(x \rightarrow y) \rightarrow((y \rightarrow z) \rightarrow(x \rightarrow z))=1$ 
(iv) $\left(x^{-} \rightarrow y^{-}\right) \rightarrow(y \rightarrow x)=1$.

Definition 2.2[7]. An algebra $(A, \rightarrow, \sim,-, \sim, 1)$ with the binary operations $\rightarrow ", " \sim "$ and the quasi complements " - ", " $"$ is called a pseudoWajsberg algebra if it satisfies the following axioms for all $x, y, z \in A$,

(i) (a) $1 \rightarrow x=x$

(ii) $(x \sim y) \rightarrow y=(y \sim x) \rightarrow x$

$$
=(y \rightarrow x) \sim x
$$$$
=(x \rightarrow y) \sim y
$$

(iii) (a) $(x \rightarrow y) \rightarrow((y \rightarrow z) \sim(x \rightarrow z))=1$

(b) $(x \sim y) \sim((y \sim z) \rightarrow(x \sim z))=1$

(iv) $1^{-}=1^{\sim}=0$

(v) (a) $\left(x^{-} \sim y^{-}\right) \rightarrow(y \rightarrow x)=1$

(b) $\quad\left(x^{\sim} \rightarrow y^{\sim}\right) \sim(y \sim x)=1$

(vi) $\quad\left(x \rightarrow y^{-}\right)^{\sim}=\left(y \sim x^{\sim}\right)^{-}$.

Definition 2.3[7]. An algebra $(A, \wedge, \vee, \rightarrow, \sim$, $-, \sim, 1)$ is called a lattice pseudo-Wajsberg algebras if it satisfies the following axioms for all $x, y \in A$,

(i) A partial ordering " $\leq$ " on a lattice pseudoWajsberg algebra $A$ such that $x \leq y$ if and only if $x \rightarrow y=1 \Leftrightarrow x \sim y=1$

(ii) $x \vee y=(x \rightarrow y) \sim y$

$$
\begin{aligned}
& =(y \rightarrow x) \backsim x \\
& =(x \sim y) \rightarrow y \\
& =(y \rightarrow x) \rightarrow x
\end{aligned}
$$

(iii) $x \wedge y=\left(x \sim(x \rightarrow y)^{\sim}\right)^{-}$

$$
\begin{aligned}
& =\left((x \rightarrow y) \rightarrow x^{-}\right)^{\sim} \\
& =\left(y \rightarrow(y \sim x)^{-}\right)^{\sim} \\
& =\left((y \sim x) \sim y^{\sim}\right)^{-} \\
& =\left(y \sim(y \rightarrow x)^{\sim}\right)^{-} \\
& =\left((y \rightarrow x) \rightarrow y^{-}\right)^{\sim} \\
& =\left(x \rightarrow(x \sim y)^{-}\right)^{\sim} \\
& =\left((x \sim y) \sim x^{\sim}\right)^{-} .
\end{aligned}
$$

Proposition2.4[7].In a lattice pseudo-Wajsberg algebra $(A, \longrightarrow, \sim,-, \sim, 1)$ which satisfies the following axioms for all $x, y, z \in A$,

(i) (a) $x \rightarrow x=1$

(b) $x \sim x=1$

(ii) (a) If $x \rightarrow y=1$ and $y \rightarrow x=1$, then $x=y$

(b) If $x \sim y=1$ and $y \sim x=1$, then $x=y$

(c) If $x \rightarrow y=1$ and $y \sim x=1$, then $x=y$

(iii) (a) $(x \rightarrow 1) \sim 1=1$ (b) $(x \sim 1) \rightarrow 1=1$

(iv) (a) $x \rightarrow 1=1$

(b) $x \sim 1=1$

(v) (a) If $x \rightarrow y=1$ and $y \rightarrow z=1$,

then $x \rightarrow z=1$

(b) If $x \sim y=1$ and $y \sim z=1$,

then $x \sim z=1$

(vi) (a) $x \rightarrow(y \sim x)=1$

(b) $x \sim(y \rightarrow x)=1$

(vii) $\quad x \rightarrow(y \sim z)=1 \Leftrightarrow y \sim(x \rightarrow z)=1$

(viii) (a) $(x \rightarrow y) \sim((z \rightarrow x) \rightarrow(z \rightarrow y))=1$

(b) $(x \sim y) \rightarrow((z \sim x) \sim(z \sim y))=1$

(ix) $x \rightarrow(y \sim z)=y \sim(x \rightarrow z)$.

Proposition 2.5[7].In a lattice pseudo-Wajsberg algebra $(A, \rightarrow, \sim,-, \sim, 1)$ which satisfies the following axioms for all $x, y \in A$,

(i) (a) $\left(x^{-} \sim 0\right) \rightarrow x=1$

(b) $\left(x^{\sim} \rightarrow 0\right) \sim x=1$

(ii) $0 \rightarrow x=1=0 \sim x$

(iii) (a) $x \rightarrow 0=x^{-}$

(b) $x \sim 0=x^{\sim}$

(iv) $\quad\left(x^{-}\right)^{\sim}=x=\left(x^{\sim}\right)^{-}$

(v) (a) $x^{\sim} \rightarrow y^{\sim}=y \sim x$

(b) $x^{-} \sim y^{-}=y \rightarrow x$

(vi) $\quad x^{\sim} \rightarrow y=y^{-} \sim x$

(vii) $x \leq y \Leftrightarrow y^{-} \leq x^{-} \Leftrightarrow y^{\sim} \leq x^{\sim}$

(viii) (a) $(x \rightarrow y)^{\sim}=\left(y^{\sim} \sim x^{\sim}\right)^{-}$

(b) $(x \sim y)^{-}=\left(y^{-} \rightarrow x^{-}\right)^{\sim}$.

Proposition 2.6[7].In a lattice pseudo-Wajsberg algebra $(A, \longrightarrow, \sim,-, \sim, 1)$ which satisfies the following axioms for all $x, y, z \in A$,

(i) (a) $(x \vee y)^{-}=x^{-} \wedge y^{-}$; $(x \vee y)^{\sim}=x^{\sim} \wedge y^{\sim}$

(b) $(x \wedge y)^{-}=x^{-} \vee y^{-} ;(x \wedge y)^{\sim}=x^{\sim} \vee y^{\sim}$

(ii) (a) $(x \vee y) \rightarrow z=(x \rightarrow z) \wedge(y \rightarrow z)$

(b) $(x \vee y) \backsim z=(x \sim z) \wedge(y \sim z)$

(iii)(a) $z \rightarrow(x \wedge y)=(z \rightarrow x) \wedge(z \rightarrow y)$

(b) $z \sim(x \wedge y)=(z \sim x) \wedge(z \sim y)$

(iv) (a) $(x \vee y) \rightarrow y=x \rightarrow y$

(b) $(x \vee y) \sim y=x \sim y$

(v) (a) $x \rightarrow(x \wedge y)=x \rightarrow y$

(b) $x \sim(x \wedge y)=x \sim y$

(vi) (a) $(x \rightarrow y) \vee(y \rightarrow x)=1$

(b) $(x \sim y) \vee(y \sim x)=1$

(vii)(a) $x \rightarrow(y \vee z)=(x \rightarrow y) \vee(x \rightarrow z)$

(b) $x \sim(y \vee z)=(x \sim y) \vee(x \sim z)$

(viii) (a) $(x \wedge y) \rightarrow z=(x \rightarrow z) \vee(y \rightarrow z)$

(b) $(x \wedge y) \backsim z=(x \sim z) \vee(y \sim z)$ 
(ix) $\quad(x \wedge y) \vee z=(x \vee z) \wedge(y \vee z)$

$$
\begin{aligned}
& \text { (x) (a) }(x \wedge y) \rightarrow z=(x \rightarrow y) \rightarrow(x \rightarrow z) \\
& =(y \rightarrow x) \rightarrow(y \rightarrow z) \\
& \text { (b) }(x \wedge y) \leadsto z=(x \sim y) \sim(x \sim z) \\
& =(y \leadsto x) \backsim(y \backsim z) \\
& \text { (xi) } \text { (a) } z \rightarrow(x \vee y)=(x \rightarrow y) \backsim(z \rightarrow y) \\
& =(y \rightarrow x) \sim(z \rightarrow x) \\
& \text { (b) } z \backsim(x \vee y)=(x \sim y) \rightarrow(z \sim y) \\
& =(y \sim x) \rightarrow(z \sim x) .
\end{aligned}
$$

Proposition 2.7[7].In lattice pseudo-Wajsberg algebra $(A, \longrightarrow, \sim,-, \sim, 1)$ which satisfies the following axioms for all $x \in A$,
(i) (a) $x^{\sim} \rightarrow x=x$
(b) $x^{\sim} \sim x=x$
(ii) (a) $\left(x \rightarrow x^{-}\right)^{\sim}=x$
(b) $\left(x \sim x^{\sim}\right)^{-}=x$
(iii) (a) $x \rightarrow x^{-}=x^{-}$
(b) $x \sim x^{\sim}=x^{\sim}$
(iv) (a) $x \vee x^{\sim}=1$
(b) $x \wedge x^{-}=0$
(v) (a) $x \wedge x^{\sim}=0$
(b) $x \vee x^{-}=1$

Definition 2.8[10]. A non-empty subset $I$ of Wajsberg algebra $A$ is called an ideal if it satisfies the following axioms for all $x, y \in A$,
(i) $0 \in I$
(ii) $x \in I$ and $y \leq x$ imply $y \in I$
(iii) $x, y \in I$ imply $x^{-} \rightarrow y \in I$.

Definition 2.9[13].Let $A$ be lattice pseudoWajsberg algebra. Let $F$ be non-empty subset of $A$ is called a $p$-ideal of $A$ if it satisfies the following axioms for all $x, y \in A$,

(i) $0 \in F$

(ii) $x \in F$ and $y \leq x$ imply $y \in F$

(iii) $x, y \in F$ imply $x^{-} \rightarrow y \in F$

(iv) $x, y \in F$ imply $x^{\sim} \sim y \in F$.

Proposition 2.10[11].Intersection of two $p$-ideals of lattice pseudo-Wajsberg algebra is a $p$-ideal.

Proposition 2.11[11].If $R$ is a nonempty family of $p$-ideals of a lattice pseudo-Wajsberg algebra $A$. Then $F=\bigcap R$ is also a $p$-ideal of $A$.

Definition 2.12[10]. Let $A$ be a set. A function $\mu: A \rightarrow[0,1]$ is called a fuzzy subset on $A$ for each $x \in A$, then the value of $\mu(x)$ describes a degree of membership of $x$ in $\mu$.

Definition 2.13[1].Let $\left\{\mu_{i}\right\}$ be an indexed family of fuzzy subsets, then $\left(\cup \mu_{i}\right)=\sup \left\{\mu_{i}\right\}$ and $\left(\cap \mu_{i}\right)=$ $\inf \left\{\mu_{i}\right\}$.

\section{MAIN RESULTS}

\subsection{Properties of fuzzy pseudo-ideal (fuzzy $p$ -} ideal)

In this section, we define fuzzy $p$-ideal of lattice pseudo-Wajsberg algebra and obtain some useful results with illustrations.

Definition 3.1.1. Let $A$ be lattice pseudo-Wajsberg algebra. Let $\mu$ be a non-empty fuzzy subset of $A$ is called a fuzzy $p$-ideal of $A$ if it satisfies the following axioms for all $x, y \in A$,

(i) $\mu(0) \geq \mu(x)$

(ii) If $y \leq x$ imply $\mu(y) \geq \mu(x)$

(iii) $\mu(x) \geq \min \left\{\mu\left(x^{-} \rightarrow y\right), \mu(y)\right\}$

(iv) $\mu(x) \geq \min \left\{\mu\left(x^{\sim} \sim y\right), \mu(y)\right\}$.

Example 3.1.2. Consider a set $A=\{0, a, b, 1\}$. Define a partial ordering " $\leq$ " on $A$, such that $0 \leq a \leq 1 ; 0 \leq b \leq 1$ and the binary operations " $\longrightarrow$ ", " $\sim$ " and the quasi complements " - ", " " given by the following tables (3.1), (3.2), (3.3) and (3.4).

\begin{tabular}{|c|c|}
\hline$x$ & $x^{-}$ \\
\hline 0 & 1 \\
\hline$a$ & $b$ \\
\hline$b$ & $a$ \\
\hline 1 & 0 \\
\hline
\end{tabular}

Table: 3.1

\begin{tabular}{|c|c|}
\hline$x$ & $x^{\sim}$ \\
\hline 0 & 1 \\
\hline$a$ & $b$ \\
\hline$b$ & $a$ \\
\hline 1 & 0 \\
\hline
\end{tabular}

Table: 3.3

Complement
Complement

\begin{tabular}{|c|c|c|c|c|}
\hline$\rightarrow$ & 0 & $a$ & $b$ & 1 \\
\hline 0 & 1 & 1 & 1 & 1 \\
\hline$a$ & $b$ & 1 & 1 & 1 \\
\hline$b$ & $a$ & $b$ & 1 & 1 \\
\hline 1 & 0 & $a$ & $b$ & 1 \\
\hline
\end{tabular}

Table: 3.2

Implication

\begin{tabular}{|c|c|c|c|c|}
\hline$\sim$ & 0 & $a$ & $b$ & 1 \\
\hline 0 & 1 & 1 & 1 & 1 \\
\hline$a$ & $b$ & 1 & 1 & 1 \\
\hline$b$ & $a$ & $a$ & 1 & 1 \\
\hline 1 & 0 & $a$ & $b$ & 1 \\
\hline
\end{tabular}

Table: 3.4

Implication
Then, $\quad A=(A, \wedge, \vee, \longrightarrow, \sim, 0,1)$ is a lattice pseudo-Wajsberg algebra and consider the fuzzy subsets $\mu_{1}$ and $\mu_{2}$ on $A$ as

$$
\mu_{1}(x)=\left\{\begin{array}{l}
0.7 \text { if } x \in\{0, b\} \\
0.3 \text { if } x \in\{a, 1\}
\end{array}\right.
$$

Then, $\mu_{1}(x)$ is a fuzzy $p$-ideal of $A$. 
But, $\quad \mu_{2}(x)=\left\{\begin{aligned} & 0.6 \text { if } x \in\{0, a, 1\} \\ & 0.4 \text { if } x \in\{b\}\end{aligned}\right.$

Then, $\mu_{2}(x)$ is not afuzzy $p$-ideal of $A$.

Since, $\mu(b) \geq \min \left\{\mu\left(b^{-} \rightarrow a\right), \mu(a)\right\}$

$$
\begin{aligned}
\mu(b) & \geq \min \{\mu(a \rightarrow a), \mu(a)\} \\
& =0.4 \geq 0.6 .
\end{aligned}
$$

Thus, $\mu(b) \nsupseteq \min \left\{\mu\left(b^{-} \rightarrow a\right), \mu(a)\right\}$.

Example 3.1.3. Consider a set $A=\{0, a, b, c, 1\}$. Define a partial ordering " $\leq$ "on $A$, such that $0 \leq$ $a \leq b \leq c \leq 1$ and the binary operations $" \rightarrow ", " \sim "$ and quasi complements" - ", " " given by the following tables (3.5), (3.6), (3.7) and (3.8).

\begin{tabular}{|l|l|}
\hline$x$ & $x^{-}$ \\
\hline 0 & 1 \\
\hline$a$ & $c$ \\
\hline$b$ & $c$ \\
\hline$c$ & $b$ \\
\hline 1 & 0 \\
\hline
\end{tabular}

Table: 3.5 Complement

\begin{tabular}{|c|c|c|c|c|c|}
\hline$\rightarrow$ & 0 & $a$ & $b$ & $c$ & 1 \\
\hline 0 & 1 & 1 & 1 & 1 & 1 \\
\hline$a$ & $c$ & 1 & 1 & 1 & 1 \\
\hline$b$ & $c$ & $c$ & 1 & $c$ & 1 \\
\hline$c$ & $b$ & $b$ & $b$ & 1 & 1 \\
\hline 1 & 0 & $a$ & $b$ & $c$ & 1 \\
\hline
\end{tabular}

Table: 3.6 Implication

\begin{tabular}{|c|c|}
\hline$x$ & $x^{\sim}$ \\
\hline 0 & 1 \\
\hline$a$ & $b$ \\
\hline$b$ & $c$ \\
\hline$c$ & $b$ \\
\hline 1 & 0 \\
\hline
\end{tabular}

Table: 3.7 Complement

\begin{tabular}{|c|c|c|c|c|c|}
\hline$\sim$ & 0 & $a$ & $b$ & $c$ & 1 \\
\hline 0 & 1 & 1 & 1 & 1 & 1 \\
\hline$a$ & $b$ & 1 & 1 & 1 & 1 \\
\hline$b$ & $c$ & $c$ & 1 & $c$ & 1 \\
\hline$c$ & $b$ & $b$ & $b$ & 1 & 1 \\
\hline 1 & 0 & $a$ & $b$ & $c$ & 1 \\
\hline
\end{tabular}

Table: 3.8 Implication
Then, $\quad A=(A, \wedge, \vee, \rightarrow, \sim, 0,1)$ is a lattice pseudo-Wajsberg algebra and consider the fuzzy subsets $\mu_{1}$ and $\mu_{2}$ on $A$ as

$$
\mu_{1}(x)=\left\{\begin{array}{c}
0.8 \text { if } x \in\{0, a, b\} \\
0.2 \text { if } x \in\{c, 1\}
\end{array}\right.
$$

Then, $\mu_{1}(x)$ is a fuzzy $p$-ideal of $A$.

But, $\quad \mu_{2}(x)=\left\{\begin{array}{c}0.9 \text { if } x \in\{0, c\} \\ 0.1 \text { if } x \in\{a, b, 1\}\end{array}\right.$

Then, $\mu_{2}(x)$ is not a fuzzy $p$-ideal of $A$.

Since, $\mu(a) \geq \min \left\{\mu\left(a^{\sim} \sim 0\right), \mu(0)\right\}$ $\mu(a) \geq \min \{\mu(b \sim 0), \mu(0)\}=0.1 \geq 0.9$.

Thus, $\mu(a) \geq \min \left\{\mu\left(a^{\sim} \sim 0\right), \mu(0)\right\}$.
Definition 3.1.4. Let $A$ be lattice pseudo-Wajsberg algebra. A fuzzy $p$-ideal $\mu$ of $A$ is a nonempty subset of $A$ is called a fuzzy $p$-lattice ideal if it satisfies the following axioms for all $x, y \in A$,

(i)If $y \leq x$ then $\mu(y) \geq \mu(x)$

(ii) $\mu(x \vee y) \geq \min \{\mu(x), \mu(y)\}$

Example 3.1.5. Consider a set $A=\{0, a, b, 1\}$. Define a partial ordering " $\leq$ " on $A$, such that $0 \leq$ $a \leq 1 ; 0 \leq b \leq 1$ and the binary operations $" \rightarrow ", " \sim$ " and the quasi complements " - ", " " given by the following tables (3.9), (3.10), (3.11) and (3.12).

\begin{tabular}{|c|c|}
\hline$x$ & $x^{-}$ \\
\hline 0 & 1 \\
\hline$a$ & $b$ \\
\hline$b$ & $a$ \\
\hline 1 & 0 \\
\hline
\end{tabular}

\begin{tabular}{|c|c|c|c|c|}
\hline$\rightarrow$ & 0 & $a$ & $b$ & 1 \\
\hline 0 & 1 & 1 & 1 & 1 \\
\hline$a$ & $b$ & 1 & 1 & 1 \\
\hline$b$ & $a$ & $b$ & 1 & 1 \\
\hline 1 & 0 & $a$ & $b$ & 1 \\
\hline
\end{tabular}

Table: 3.9

Table: 3.10

Complement Implication

\begin{tabular}{|c|c|}
\hline$x$ & $x^{\sim}$ \\
\hline 0 & 1 \\
\hline$a$ & $b$ \\
\hline$b$ & $a$ \\
\hline 1 & 0 \\
\hline
\end{tabular}

\begin{tabular}{|l|l|l|l|l|}
\hline$\sim$ & 0 & $a$ & $b$ & 1 \\
\hline 0 & 1 & 1 & 1 & 1 \\
\hline$a$ & $b$ & 1 & 1 & 1 \\
\hline$b$ & $a$ & $a$ & 1 & 1 \\
\hline 1 & 0 & $a$ & $b$ & 1 \\
\hline
\end{tabular}

Table: 3.11

Tables: $\mathbf{3 . 1 2}$

Complement Implication

Then, $\quad A=(A, \wedge, \vee, \rightarrow, \sim, 0,1)$ is a lattice pseudo-Wajsberg algebra and consider the fuzzy subsets $\mu_{1}$ and $\mu_{2}$ on $A$ as

$$
\mu_{1}(x)=\left\{\begin{array}{l}
0.5 \text { if } x \in\{0, b\} \\
0.3 \text { if } x \in\{a, 1\}
\end{array}\right.
$$

Then, $\mu_{1}(x)$ is a fuzzy $p$-lattice ideal of $A$.

But, $\quad \mu_{2}(x)=\left\{\begin{array}{r}0.8 \text { if } x \in\{0, a, b\} \\ 0.2 \text { if } x \in\{1\}\end{array}\right.$ 
Then, $\mu_{2}(x)$ is not a fuzzy $p$-lattice ideal of $A$, [from (ii) of definition 2.3]

Since, $\mu(a \vee b)=\mu((b \sim a) \rightarrow a)=\mu((a \rightarrow a))$ $=\mu(1) \geq \min \{\mu(a), \mu(b)\}$

$=0.2 \geq 0.8$.

Thus, $\mu(a \vee b) \geq \min \{\mu(a), \mu(b)\}$.

Proposition 3.1.6. Let $A$ be lattice pseudoWajsberg algebra. Every fuzzy $p$-ideal of $A$ is a fuzzy $p$-lattice ideal.

Proof. Let $\mu$ be a fuzzy $p$-ideal of $A$. From (ii) of definition 3.1.1 shows that $\mu$ satisfies (i) of definition 3.1.4, for any $x, y \in A$ then,

$$
\begin{aligned}
& (x \vee y)^{-} \rightarrow y=((x \rightarrow y) \sim y)^{-} \rightarrow y \\
& =x^{-} \rightarrow y \leq\left(x^{-}\right)^{\sim}=x \\
& (x \vee y)^{\sim} \sim y=((x \sim y) \rightarrow y)^{\sim} \sim y \\
& =x^{\sim} \sim y \leq\left(x^{\sim}\right)^{-}=x \\
& \min \left\{\mu\left((x \vee y)^{-} \rightarrow y\right), \mu(y)\right\} \\
& =\min \left\{\mu\left(((x \rightarrow y) \sim y)^{-} \rightarrow y\right), \mu(y)\right\} \\
& =\min \left\{\mu\left(x^{-} \rightarrow y\right), \mu(y)\right\} \\
& \quad \leq \min \left\{\mu\left(\left(x^{-}\right)^{\sim}\right), \mu(y)\right\} \\
& \quad \leq \min \{\mu(x), \mu(y)\} \leq \mu(x) \\
& \min \left\{\mu\left((x \vee y)^{\sim} \sim y\right), \mu(y)\right\} \\
& =\min \left\{\mu\left(((x \sim y) \rightarrow y)^{\sim} \sim y\right), \mu(y)\right\} \\
& =\min \left\{\mu\left(x^{\sim} \sim y\right), \mu(y)\right\} \\
& \leq \min \left\{\mu\left(\left(x^{\sim}\right)^{-}\right), \mu(y)\right\} \\
& \leq \min \{\mu(x), \mu(y)\} \leq \mu(x)
\end{aligned}
$$

It is enough to prove

$$
\begin{aligned}
\mu(x \vee y) & \geq \min \{\mu(x), \mu(y)\} \\
\mu(x \vee y) & \geq \min \left\{\mu\left((x \vee y)^{-} \rightarrow y\right), \mu(y)\right\} \\
& \geq \min \left\{\mu\left((x \vee y)^{\sim} \sim y\right), \mu(y)\right\} \\
& \geq \min \{\mu(x), \mu(y)\}
\end{aligned}
$$

Hence, $\mu$ is a fuzzy $p$-lattice ideal of $A$.

Remark 3.1.7. The converse of Proposition 3.1 .6 may be true. In Example 3.1.5, $\mu$ is a fuzzy subset of $A$ defined by

$\mu(x)=\left\{\begin{array}{c}0.7 \text { if } x \in\{0, b\} \\ 0.4 \text { if } x \in\{a, 1\}\end{array} \quad\right.$ then $\mu$ is a fuzzy $p$-lattice ideal and also $\mu$ is a fuzzy $p$-idealof $A$.

Proposition 3.1.8. The intersection of any set of fuzzy $p$-ideals in lattice-pseudo Wajsberg algebra is also a fuzzy $p$-ideal.
Proof. Let $\left\{\mu_{i}\right\}$ be a family of fuzzy $p$-ideals of lattice-pseudo Wajsberg algebra $A$ then for all $x, y \in A$,

$$
\begin{aligned}
&\left(\cap \mu_{i}\right)(0)=\operatorname{Inf}\left\{\mu_{i}(0)\right\} \geq \operatorname{Inf}\left\{\mu_{i}(x)\right\} \geq\left(\cap \mu_{i}\right)(x) . \\
& \operatorname{Let}\left(\cap \mu_{i}\right)(x)=\operatorname{Inf}\left\{\mu_{i}(x)\right\} \\
& \geq \operatorname{Inf}\left\{\min \left\{\mu_{i}\left(x^{-} \rightarrow y\right), \mu_{i}(y)\right\}\right\} \\
& \geq \min \left\{\operatorname{Inf}\left(\mu_{i}\left(x^{-} \rightarrow y\right)\right), \operatorname{Inf}\left(\mu_{i}(y)\right)\right\} \\
& \geq \min \left\{\left(\cap \mu_{i}\right)\left(x^{-} \rightarrow y\right),\left(\cap \mu_{i}\right)(y)\right\}
\end{aligned}
$$

Therefore,

$\left(\cap \mu_{i}\right)(x) \geq \min \left\{\left(\cap \mu_{i}\right)\left(x^{-} \rightarrow y\right),\left(\cap \mu_{i}\right)(y)\right\}$ and $\operatorname{also}\left(\cap \mu_{i}\right)(x)=\operatorname{Inf}\left\{\mu_{i}(x)\right\}$

$$
\begin{aligned}
& \geq \operatorname{Inf}\left\{\min \left\{\mu_{i}\left(x^{\sim} \sim y\right), \mu_{i}(y)\right\}\right\} \\
& \geq \min \left\{\operatorname{Inf}\left(\mu_{i}\left(x^{\sim} \sim y\right)\right), \operatorname{Inf}\left(\mu_{i}(y)\right)\right\} \\
& \geq \min \left\{\left(\bigcap \mu_{i}\right)\left(x^{\sim} \sim y\right),\left(\bigcap \mu_{i}\right)(y)\right\}
\end{aligned}
$$

Therefore,

$$
\left(\cap \mu_{i}\right)(x) \geq \min \left\{\left(\cap \mu_{i}\right)\left(x^{\sim} \sim y\right),\left(\cap \mu_{i}\right)(y)\right\}
$$

Hence, the intersection of any set of fuzzy $p$-ideals in lattice-pseudo Wajsberg algebra is also fuzzypideal.

\subsection{Properties of Anti-fuzzy pseudo-ideal (anti- fuzzy $p$-ideal)}

In this section, we define anti-fuzzy $p$-ideal of lattice pseudo-Wajsberg algebra and obtain some useful results with illustrations.

Definition 3.2.1. Let $A$ be lattice pseudo-Wajsberg algebra. Let $\mu$ be a non-empty fuzzy subset of $A$ is called an anti-fuzzy $p$-ideal of $A$ if it satisfies the following axioms for all $x, y \in A$,

(i) $\mu(0) \leq \mu(x)$

(ii) If $y \leq x$ imply $\mu(y) \leq \mu(x)$

(iii) $\mu(x) \leq \max \left\{\mu\left(x^{-} \rightarrow y\right), \mu(y)\right\}$

(iv) $\mu(x) \leq \max \left\{\mu\left(x^{\sim} \sim y\right), \mu(y)\right\}$.

Example3.2.2. In Example 3.1.2, consider the fuzzy subsets $\mu_{1}$ and $\mu_{2}$ on $A$ as

$$
\mu_{1}(x)=\left\{\begin{array}{l}
0.2 \text { if } x \in\{0, b\} \\
0.8 \text { if } x \in\{a, 1\}
\end{array}\right.
$$

Then, $\mu_{1}(x)$ is an anti-fuzzy $p$-ideal of $A$.

But, $\quad \mu_{2}(x)=\left\{\begin{aligned} 0.3 \text { if } x \in\{0, a, 1\} \\ 0.7 \text { if } x \in\{b\}\end{aligned}\right.$

Then, $\mu_{2}(x)$ is not an anti-fuzzy $p$-ideal of $A$.

Since, $\mu(b) \leq \max \left\{\mu\left(b^{-} \rightarrow a\right), \mu(a)\right\}$ 


$$
\begin{aligned}
\mu(b) & \leq \max \{\mu(a \rightarrow a), \mu(a)\} \\
& =0.7 \nsubseteq 0.3 .
\end{aligned}
$$

Thus, $\mu(b) \$ \max \left\{\mu\left(b^{-} \rightarrow a\right), \mu(a)\right\}$.

Example 3.2.3. In Example 3.1.3, consider the fuzzy subsets $\mu_{1}$ and $\mu_{2}$ on $A$ as

$$
\mu_{1}(x)=\left\{\begin{array}{c}
0.3 \text { if } x \in\{0, a, b\} \\
0.7 \text { if } x \in\{c, 1\}
\end{array}\right.
$$

Then, $\mu_{1}(x)$ is an anti-fuzzy $p$-ideal of $A$.

But, $\quad \mu_{2}(x)=\left\{\begin{array}{l}0.4 \text { if } x \in\{0, c\} \\ 0.6 \text { if } x \in\{a, b, 1\}\end{array}\right.$

Then, $\mu_{2}(x)$ is not an anti-fuzzy $p$-ideal of $A$.

Since, $\mu(a) \leq \max \left\{\mu\left(a^{\sim} \sim 0\right), \mu(0)\right\}$

$$
\mu(a) \leq \max \{\mu(b \sim 0), \mu(0)\}=0.6 \$ 0.4 .
$$

Thus, $\mu(a) \$ \max \left\{\mu\left(a^{\sim} \sim 0\right), \mu(0)\right\}$.

Definition 3.2.4. Let $A$ be lattice pseudo-Wajsberg algebra. An anti-fuzzy $p$-ideal $\mu$ of $A$ is a nonempty subset of Ais called an anti-fuzzy $p$-lattice ideal if it satisfies the following axioms for all $x, y \in A$,

(i)If $y \leq x$ then $\mu(y) \leq \mu(x)$

(ii) $\mu(x \vee y) \leq \max \{\mu(x), \mu(y)\}$.

Example 3.2.5. In Example 3.1.5, consider the fuzzy subsets $\mu_{1}$ and $\mu_{2}$ on $A$ as

$$
\mu_{1}(x)=\left\{\begin{array}{l}
0.3 \text { if } x \in\{0, b\} \\
0.5 \text { if } x \in\{a, 1\}
\end{array}\right.
$$

Then, $\mu_{1}(x)$ is an anti-fuzzy $p$-lattice ideal of $A$.

But, $\quad \mu_{2}(x)=\left\{\begin{array}{r}0.2 \text { if } x \in\{0, a, b\} \\ 0.6 \text { if } x \in\{1\}\end{array}\right.$

Then, $\mu_{2}(x)$ is not an anti-fuzzy $p$-lattice ideal of $A$, [from (ii) of definition 2.3]

Since, $\mu(a \vee b)=\mu((b \sim a) \rightarrow a)=\mu((a \rightarrow a))$

$$
\begin{aligned}
& =\mu(1) \leq \max \{\mu(a), \mu(b)\} \\
& =0.6 \pm 0.2 .
\end{aligned}
$$

Thus, $\mu(a \vee b) \$ \max \{\mu(a), \mu(b)\}$.

Proposition 3.2.6. Let $A$ be lattice pseudoWajsberg algebra. Every anti-fuzzy $p$-ideal of $A$ is an anti-fuzzyp-lattice ideal.

Proof. Let $\mu$ be an anti-fuzzyp-ideal of $A$. From (ii) of definition 3.2.1 shows that $\mu$ satisfies (i) of definition 3.2.4, for any $x, y \in A$ then,

$$
\begin{aligned}
(x \vee y)^{-} \rightarrow y & =((x \rightarrow y) \sim y)^{-} \rightarrow y \\
& =x^{-} \rightarrow y \leq\left(x^{-}\right)^{\sim}=x \\
(x \vee y)^{\sim} \sim y & =((x \sim y) \rightarrow y)^{\sim} \sim y \\
& =x^{\sim} \sim y \leq\left(x^{\sim}\right)^{-}=x \\
\max \left\{\mu \left((x \vee y)^{-}\right.\right. & \rightarrow y), \mu(y)\}
\end{aligned}
$$

$$
\begin{aligned}
&=\max \left\{\mu\left(((x \rightarrow y) \sim y)^{-} \rightarrow y\right), \mu(y)\right\} \\
&=\max \left\{\mu\left(x^{-} \rightarrow y\right), \mu(y)\right\} \\
& \geq \max \left\{\mu\left(\left(x^{-}\right)^{\sim}\right), \mu(y)\right\} \\
& \geq \max \{\mu(x), \mu(y)\} \geq \mu(x) \\
& \max \left\{\mu\left((x \vee y)^{\sim} \sim y\right), \mu(y)\right\} \\
& \quad=\max \left\{\mu\left(((x \sim y) \rightarrow y)^{\sim} \sim y\right), \mu(y)\right\} \\
& \quad=\max \left\{\mu\left(x^{\sim} \sim y\right), \mu(y)\right\} \\
& \quad \geq \max \left\{\mu\left(\left(x^{\sim}\right)^{-}\right), \mu(y)\right\} \\
& \geq \max \{\mu(x), \mu(y)\} \geq \mu(x)
\end{aligned}
$$

It is enough to prove

$$
\begin{aligned}
\mu(x \vee y) & \leq \max \{\mu(x), \mu(y)\} \\
\mu(x \vee y) & \leq \max \left\{\mu\left((x \vee y)^{-} \rightarrow y\right), \mu(y)\right\} \\
& \leq \max \left\{\mu\left((x \vee y)^{\sim} \sim y\right), \mu(y)\right\} \\
& \leq \max \{\mu(x), \mu(y)\}
\end{aligned}
$$

Hence, $\mu$ is an anti-fuzzy $p$-lattice ideal of $A$.

3.3 Properties of Intuitionistic fuzzy pseudoideal (Intuitionistic fuzzy $\boldsymbol{p}$-ideal)

In this section, we define an intuitionistic fuzzypidealof lattice pseudo-Wajsberg algebra and obtain some results with illustrations.

Definition 3.3.1. Let $A$ be a lattice pseudoWajsberg algebra. An intuitionistic fuzzy subset $K=\left(\alpha_{K}, \beta_{K}\right)$ of $A$ is called an intuitionisticfuzzy $p$-ideal of $A$ if it satisfies the following for all $x, y \in A$,

(i) (a) $\alpha_{K}(0) \geq \alpha_{K}(x)$

(b) $\beta_{K}(0) \leq \beta_{K}(x)$

(ii) If $y \leq x$ imply $\alpha_{K}(x) \geq \alpha_{K}(y)$ and

$$
\beta_{K}(x) \leq \beta_{K}(y) \text {. }
$$

(iii) (a) $\alpha_{K}(x) \geq \min \left\{\alpha_{K}\left(x^{-} \rightarrow y\right), \alpha_{K}(y)\right\}$

(b) $\beta_{K}(x) \leq \max \left\{\beta_{K}\left(x^{-} \rightarrow y\right), \beta_{K}(y)\right\}$

(iv) (a) $\alpha_{K}(x) \geq \min \left\{\alpha_{K}\left(x^{\sim} \sim y\right), \alpha_{K}(y)\right\}$

(b) $\beta_{K}(x) \leq \max \left\{\beta_{K}\left(x^{\sim} \sim y\right), \beta_{K}(y)\right\}$.

Example3.3.2. In Example 3.1.2, consider an intuitionistic fuzzy subset $K=\left(\alpha_{K}, \beta_{K}\right)$ of $A$

as,

$$
\begin{aligned}
& \alpha_{K}(x)=\left\{\begin{array}{l}
0.7 \text { if } x \in\{0, b\} \\
0.3 \text { if } x \in\{a, 1\}
\end{array}\right. \\
& \beta_{K}(x)=\left\{\begin{array}{l}
0.2 \text { if } x \in\{0, b\} \\
0.8 \text { if } x \in\{a, 1\}
\end{array}\right.
\end{aligned}
$$


Then, $K$ is an intuitionistic fuzzy $p$-ideal of $A$. But, $F=\left(\alpha_{F}, \beta_{F}\right)$ is an intuitionistic fuzzy subset on $A$ as, $\quad \alpha_{F}(x)=\left\{\begin{aligned} 0.6 & \text { if } x \in\{0, a, 1\} \\ 0.4 & \text { if } x \in\{b\}\end{aligned}\right.$

$$
\beta_{F}(x)=\left\{\begin{aligned}
0.3 & \text { if } x \in\{0, a, 1\} \\
0.7 & \text { if } x \in\{b\}
\end{aligned}\right.
$$

Then, $F$ is not an intuitionistic fuzzy $p$-ideal of $A$.

Since, $\alpha_{F}(b) \geq \min \left\{\alpha_{F}\left(b^{-} \rightarrow a\right), \alpha_{F}(a)\right\}$

$$
\begin{aligned}
& \geq \min \left\{\alpha_{F}(a \rightarrow a), \alpha_{F}(a)\right\} \\
& =0.4 \geq 0.6 .
\end{aligned}
$$

Thus, $\alpha_{F}(b) \geq \min \left\{\alpha_{F}\left(b^{-} \rightarrow a\right), \mu(a)\right\}$.

Since, $\beta_{F}(b) \leq \max \left\{\beta_{F}\left(b^{-} \rightarrow a\right), \beta_{F}(a)\right\}$

$$
\begin{aligned}
& \leq \max \left\{\beta_{F}(a \rightarrow a), \beta_{F}(a)\right\} \\
& =0.7 \nsubseteq 0.3 .
\end{aligned}
$$

Thus, $\beta_{F}(b) \$ \max \left\{\beta_{F}\left(b^{-} \rightarrow a\right), \beta_{F}(a)\right\}$.

Definition 3.3.3.Let $A$ be lattice pseudo-Wajsberg algebra. A intuitionistic fuzzy $p$-ideal $\mu$ of $A$ is a nonempty subset of $A$ is called anintuitionistic fuzzy $p$-lattice ideal if it satisfies the following axioms for all $x, y \in A$,

(i) If $y \leq x$ then $\alpha_{K}(y) \geq \alpha_{K}(x)$ and

$$
\beta_{K}(y) \leq \beta_{K}(x)
$$

(ii) $\alpha_{K}(x \vee y) \geq \min \left\{\alpha_{K}(x), \alpha_{K}(y)\right\}$

(iii) $\beta_{K}(x \vee y) \leq \max \left\{\beta_{K}(x), \beta_{K}(y)\right\}$.

Example3.3.4. In Example 3.1.5, consider an intuitionistic fuzzy subsets $K=\left(\alpha_{K}, \beta_{K}\right)$ of $A$ as

$$
\begin{aligned}
& \alpha_{K}(x)=\left\{\begin{array}{l}
0.5 \text { if } x \in\{0, b\} \\
0.3 \text { if } x \in\{a, 1\}
\end{array}\right. \\
& \beta_{K}(x)=\left\{\begin{array}{l}
0.3 \text { if } x \in\{0, b\} \\
0.5 \text { if } x \in\{a, 1\}
\end{array}\right.
\end{aligned}
$$

Then, $K$ is an intuitionistic fuzzy $p$-lattice ideal of A.

But, $F=\left(\alpha_{F}, \beta_{F}\right)$ is an intuitionistic fuzzy subset on $A$ as, $\alpha_{F}(x)=\left\{\begin{aligned} 0.8 & \text { if } x \in\{0, a, b\} \\ 0.2 & \text { if } x \in\{1\}\end{aligned}\right.$

$$
\beta_{F}(x)=\left\{\begin{aligned}
0.2 & \text { if } x \in\{0, a, b\} \\
0.6 & \text { if } x \in\{1\}
\end{aligned}\right.
$$

Then, $F$ is not an intuitionistic fuzzyp-lattice ideal of $A, \quad$ [from (ii) of definition 2.3]

Since,

$$
\begin{aligned}
\alpha_{F}(a \vee b) & =\alpha_{F}((b \sim a) \rightarrow a)=\alpha_{F}((a \rightarrow a)) \\
& =\alpha_{F}(1) \geq \min \left\{\alpha_{F}(a), \alpha_{F}(b)\right\} \\
& =0.1 \geq 0.8 . \\
\beta_{F}(a \vee b) & =\beta_{F}((b \sim a) \rightarrow a)=\beta_{F}((a \rightarrow a))
\end{aligned}
$$

$$
\begin{aligned}
& =\beta_{F}(1) \leq \max \left\{\beta_{F}(a), \beta_{F}(b)\right\} \\
& =0.7 \$ 0.2 .
\end{aligned}
$$

Thus, $\alpha_{F}(a \vee b) \nsupseteq \min \left\{\alpha_{F}(a), \alpha_{F}(b)\right\}$ and

$$
\beta_{F}(a \vee b) \$ \max \left\{\beta_{F}(a), \beta_{F}(b)\right\} \text {. }
$$

Proposition 3.3.5. Let $A$ be lattice pseudoWajsberg algebra. Every intuitionistic fuzzy $p$-ideal of $A$ is an intuitionistic fuzzyp-lattice ideal.

Proof. Let $\mu$ be an intuitionistic fuzzy $p$-ideal of $A$. From (ii) of definition 3.3.1 shows that $\mu$ satisfies (i) of definition 3.3.3. for any $x, y \in A$ then,

$$
\begin{aligned}
(x \vee y)^{-} \rightarrow y & =((x \rightarrow y) \sim y)^{-} \rightarrow y \\
& =x^{-} \rightarrow y \leq\left(x^{-}\right)^{\sim}=x \\
(x \vee y)^{\sim} \sim y & =((x \sim y) \rightarrow y)^{\sim} \sim y \\
& =x^{\sim} \sim y \leq\left(x^{\sim}\right)^{-}=x
\end{aligned}
$$

$\min \left\{\alpha_{K}\left((x \vee y)^{-} \rightarrow y\right), \alpha_{K}(y)\right\}$

$$
\begin{aligned}
& =\min \left\{\alpha_{K}\left(((x \rightarrow y) \sim y)^{-} \rightarrow y\right), \alpha_{K}(y)\right\} \\
& =\min \left\{\alpha_{K}\left(x^{-} \rightarrow y\right), \alpha_{K}(y)\right\} \\
& \leq \min \left\{\alpha_{K}\left(\left(x^{-}\right)^{\sim}\right), \alpha_{K}(y)\right\} \\
& \leq \min \left\{\alpha_{K}(x), \alpha_{K}(y)\right\} \leq \alpha_{K}(x)
\end{aligned}
$$

$\max \left\{\beta_{K}\left((x \vee y)^{-} \rightarrow y\right), \beta_{K}(y)\right\}$

$$
\begin{aligned}
& =\max \left\{\beta_{K}\left(((x \rightarrow y) \sim y)^{-} \rightarrow y\right), \beta_{K}(y)\right\} \\
& =\max \left\{\beta_{K}\left(x^{-} \rightarrow y\right), \beta_{K}(y)\right\} \\
& \geq \max \left\{\beta_{K}\left(\left(x^{-}\right)^{\sim}\right), \beta_{K}(y)\right\} \\
& \geq \max \left\{\beta_{K}(x), \beta_{K}(y)\right\} \geq \beta_{K}(x)
\end{aligned}
$$$$
\min \left\{\alpha_{K}\left((x \vee y)^{\sim} \sim y\right), \alpha_{K}(y)\right\}
$$$$
=\min \left\{\alpha_{K}\left(((x \sim y) \rightarrow y)^{\sim} \sim y\right), \alpha_{K}(y)\right\}
$$$$
=\min \left\{\alpha_{K}\left(x^{\sim} \sim y\right), \alpha_{K}(y)\right\}
$$$$
\leq \min \left\{\alpha_{K}\left(\left(x^{\sim}\right)^{-}\right), \alpha_{K}(y)\right\}
$$$$
\leq \min \left\{\alpha_{K}(x), \alpha_{K}(y)\right\} \leq \alpha_{K}(x)
$$$$
\max \left\{\beta_{K}\left((x \vee y)^{\sim} \sim y\right), \beta_{K}(y)\right\}
$$$$
=\max \left\{\beta_{K}\left(((x \sim y) \rightarrow y)^{\sim} \sim y\right), \beta_{K}(y)\right\}
$$$$
=\max \left\{\beta_{K}\left(x^{\sim} \sim y\right), \beta_{K}(y)\right\}
$$$$
\geq \max \left\{\beta_{K}\left(\left(x^{\sim}\right)^{-}\right), \beta_{K}(y)\right\}
$$$$
\geq \max \left\{\beta_{K}(x), \beta_{K}(y)\right\} \geq \beta_{K}(x)
$$

It is enough to prove

$$
\begin{aligned}
\alpha_{K}(x \vee y) & \geq \min \left\{\alpha_{K}(x), \alpha_{K}(y)\right\} \\
\alpha_{K}(x \vee y) & \geq \min \left\{\alpha_{K}\left((x \vee y)^{-} \rightarrow y\right), \alpha_{K}(y)\right\} \\
& \geq \min \left\{\alpha_{K}\left((x \vee y)^{\sim} \sim y\right), \alpha_{K}(y)\right\}
\end{aligned}
$$




$$
\begin{aligned}
& \geq \min \left\{\alpha_{K}(x), \alpha_{K}(y)\right\} \\
\beta_{K}(x \vee y) & \leq \max \left\{\beta_{K}(x), \beta_{K}(y)\right\} \\
\beta_{K}(x \vee y) & \leq \max \left\{\beta_{K}\left((x \vee y)^{-} \rightarrow y\right), \beta_{K}(y)\right\} \\
& \leq \max \left\{\beta_{K}\left((x \vee y)^{\sim} \sim y\right), \beta_{K}(y)\right\} \\
& \leq \max \left\{\beta_{K}(x), \beta_{K}(y)\right\}
\end{aligned}
$$

Hence, $\mu$ is an intuitionistic fuzzy $p$-lattice ideal of A.

\section{CONCLUSION}

In the present paper, we have introduced the notions of fuzzy $p$-ideal, anti-fuzzy $p$-ideal, intuitionistic fuzzy $p$-ideal and fuzzy $p$-lattice ideal, anti-fuzzy $p$-lattice ideal and intuitionistic fuzzy $p$ lattice ideal in lattice pseudo-Wajsberg algebra. Also, we have discussed some of their related properties with illustrations.

\section{REFERENCES}

[1] Afroja Akter., Abeda Sultana., Abdul Alim., Supremum and Infimum Operations on Fuzzy Sets, Journal of Progressive Research in Mathematics, Volume 4, Issue 3, (2015), 366371.

[2] Azriel Rosenfeld, Fuzzy Groups, Journal of Mathematics Analysis and Applications, 35, (1971), 512-517.

[3] Biswas, R. Fuzzy subgroups and anti-fuzzy subgroups, Fuzzy Sets and systems, 35, (1990), 121-124.

[4] Ceterchi Rodica., Pseudo-Wajsberg Algebras, Multi. Valued. Logic, Volume 6, (2001), 6788.
[5] CeterchiRodica., The Lattice Structure of Pseudo-Wajsberg Algebras, Journal of Universal Computer Science, Volume.6, Number.1, (2000), 22-38.

[6] Font, J. M., Rodriguez, A. J., and Torrens, A., Wajsberg algebras, STOCHASTICA, Volume 8, Number 1, (1984), 5-31.

[7] Ibrahim, A., Indhumathi, M., PWI-Ideals of Lattice Pseudo-Wajsberg algebras, Advances in Theoretical and Applied Mathematics, Volume 13, Number 1, (2018), 1-14.

[8] Ibrahim, A., Indhumathi, M., Fuzzy PWIIdeals of Lattice Pseudo-Wajsberg algebras, International Journal of Mathematics and its Applications, Volume 6, Number 4, (2018), 21-31.

[9] Ibrahim, A., Indhumathi, M., P-Ideals of Lattice Pseudo-Wajsberg algebras, Mantech Publication, Journal of Engineering Mathematics and Statistics, Volume 3, Issue 1, (2018), 1-10.

[10] Ibrahim, A., Indhumathi, M., Anti-fuzzy PWIIdeals of Lattice Pseudo-Wajsberg algebras, Journal of Computer and Mathematical Sciences, Volume 9, Issue 8, (2018), 903-913.

[11] Ibrahim, A., Indhumathi, M., Intuitionistic fuzzy PWI-Ideals of Lattice Pseudo-Wajsberg algebras, Journal of Applied Science and Computations , Volume 5, Issue 10, (2018), 1271-1282.

[12] Wajsberg, M., Beiträge zum Metaaussagenkalkül I, Monat. Mat. Phys. 42, (1935), 221-242.

[13]Zadeh, L. A., Fuzzy sets, Inform. Control 8 (1965), 338-353. 